\section{THE TREATMENT OF TUBERCULOUS EMPYEMA *}

\section{BY}

M. P. SUSMAN, M.B., CH.M., F.R.C.S.

HONORARY ASSISTANT SURGEON, SYDNEY HOSPITAL, AND HONORARY THORACIC SURGEON, MATER MISERICORDIAE HOSPITAL, SYDNEY

In discussing the treatment of any particular tuberculous lesion it is necessary to stress a commonplace-namely, that we are dealing with a tuberculous patient and not just a local focus; constitutional treatment is therefore essential throughout, and this includes all those dietetic, hygienic, and medicinal measures that have stood the test of time.

A second point to remember is that, although a certain amount of standardization is necessary and desirable, each patient should receive individual treatment. This is attained by correlating and adapting the principles of treatment discussed below to the special features peculiar to every patient.

\section{Methods and Principles of Treatment}

Minor surgical procedures are usually done by a physician. They comprise: (1) aspiration alone, or with air replacement and/or irrigation; and (2) oleothorax. Major surgical operations consist of : (1) intercostal drainage, open or closed ; (2) thoracotomy and drainage, open or closed ; (3) phrenic avulsion, either alone, or to supplement thoracoplasty or pneumothorax or oleothorax ; (4) thoracoplasty, partial or complete ; and (5) rib resection and excision of granulation tissue and thickened pleura forming the outer wall of the cavity, or the fashioning of a hinged flap from this tissue, which is then brought into close contact with the visceral pleura.

The principles of treatment may be divided into general (in addition to constitutional treatment) and particular. Under the former heading comes: (1) the avoidance of secondary infection, or, if it occurs, its prompt and adequate treatment to minimize constitutional and local damage ; and (2) the relief of symptoms, especially those due to mechanical causes, such as a large collection of fluid or a valvular pneumothorax. In the group labelled "particular" two possibilities have to be considered: (1) if the underlying lung is apparently healthy we aim to obliterate the empyema cavity by getting the lung to expand ; (2) if the lung is diseased, and especially if there is cavitation, we must treat the pulmonary lesion as well as the empyema, so that we aim to keep the lung collapsed.

\section{Choice of Treatment}

It is clear that the main factors that determine the treatment to be adopted are: $(a)$ the state of the lung or lungs; $(b)$ the presence or absence of pyogenic infection. Subsidiary factors include: the age and general condition of the patient, the age of the empyema, the presence of bronchial or cutaneous fistula, the presence of tuberculosis elsewhere, and the severity of the infection and the response to preliminary treatment.

Since the condition of the lung and secondary infection are the most important modifying factors, it is convenient to describe the details of treatment under the following four headings:

(A) There are no signs of pulmonary tuberculosis and there are no pyogenic organisms in the empyema.

(B) Pulmonary tuberculosis is known to be present, but there are no pyogenic organisms in the empyema.

* Read in opening a discussion in the Section of Public Medicine at the Annual Meeting of the British Medical Association, Melbourne, 1935 .
(C) There are no signs of pulmonary tuberculosis, but pyogenic organisms are present in the empyema.

(D) Pulmonary tuberculosis is known to be present, and pyogenic organisms are present in the empyema.

There are several points to make about these groups. Though we say that there is no evidence of pulmonary tuberculosis in Groups $\mathrm{A}$ and $\mathrm{C}$, many of these patients do have a lung lesion, even if it only a small one. The number of patients in Groups $A$ and $C$ is very much less than the number in Groups $B$ and $D$. The absence of pyogenic organisms on one or two examinations of the pleural fluid is not enough to exclude secondary infection; repeated smears and cultures must be made when there is doubt. At the same time the patient should not, as a rule, be treated as though he had a secondary infection until the pyogenic germs are found. The absolute diagnosis of tuberculous empyema depends on the finding of the specific bacillus or on a positive animal inoculation; less certain criteria are the microscopical appearances of a piece of the pleura or the wall of a sinus, the seeing of tubercles through a thoracoscope, and an excess of lymphocytes in the pleural fluid. All these tests may be negative on one or two occasions, so that many examinations may be necessary. Finally, a tuberculous empyema often begins as a serous effusion in one of several ways. It may complicate: (1) an artificial pneumothorax, (2) a spontaneous pneumothorax, (3) pulmonary tuberculosis without any obvious cause ; (4) it may be due to rupture or puncture of a subpleural focus, or to rupture or division of pleural adhesions ; (5) it may arise apparently de novo, without any obvious tuberculosis of the lung (Groups A and C). This, I repeat, is rare, although pleurisy with serous effusion that does not become an empyema is common enough.

\section{Treatment of Group A}

(NO KNOWN LUNG LESION AND NO PYOGENIC INFECTION)

Our object is to free the pleural cavity of pus and to secure obliteration of the cavity by getting the lung to expand. Aspiration alone may suffice, but it is usually necessary to supplement this by air replacement in decreasing amounts, thus allowing the lung to creep out, an injection of $5 \mathrm{c} . \mathrm{cm}$. of methylene-blue (2 per cent.) being made to sterilize the effusion and act as pleural lavage. Alternatively, the lung may expand to a certain limit and stop: a local thoracoplasty over the residual cavity may be enough to obliterate it. Should the lung fail to expand at all, and fluid persistently re-form after aspirations, a complete thoracoplasty in two or more stages is required. Between the stages any pus should be aspirated, otherwise it may rupture into a bronchus or cause pressure symptoms as well as prevent the two pleural surfaces from adhering. The great thing is to get the thoracoplasty done before secondary infection occurs.

Finally, if the empyema is an old one, and the outer wall is a thick layer of organizing granulation tissue, it may be necessary to excise this wall or make a flap from it.

\section{Treatment of Group B}

(LUNG LESION PRESENT BUT NO PYOGENIC INFECTION)

Here our aim is to cure the empyema and at the same time to treat the underlying lung disease by keeping the lung collapsed. Instead of putting in decreasing amounts of air, as in Group A, we put in amounts sufficient to keep the lung down. If the lung tends to expand in spite of the air refills, or effusion persistently recurs, oleothorax should be tried. If the latter fails we do a thoracoplasty with phrenic avulsion. If thoracoplasty is contraindicated by the patient's general condition or by disease in the opposite lung, intercostal drainage and 
lavage may tide him over until he is fit for thoracoplasty; if he does not improve enough, the drainage must be permanent.

An exception to these rules must, however, be noted. If the tuberculous empyema follows artificial pneumothorax late in the course of the treatment, the lung may have recovered so much that we may safely allow it to expand, as in Group A.

\section{Treatment of Group C}

(NO KNOWN LUNG LESION, BUT PYOGENIC INFECTION PRESENT)

Our objective is the same as in Group A, but often the secondary infection is too severe for the simpler methods of treatment. If the patient's condition is good and the infection is mild, aspiration, air replacement, and irrigation may lead to cure. Usually the above treatment only brings improvement, and then we should institute closed intercostal drainage plus irrigation if there is no bronchial fistula. Oleothorax may be tried before drainage, but the results are variable; it may succeed in closing a small bronchial fistula. Where there is a large bronchial fistula rib resection and open drainage is the best treatment. Very rarely the fistula closes and the lung re-expands. Most of these patients need a complete thoracoplasty, with or withuit excision of the outer wall of the cavity, or conversion of it into a flap. Neither cutaneous fistula nor bronchial fistula is a contraindication; both may, in fact, be cured by the operation. The severity of the secondary infection must be first reduced as far as possible by frequent irrigations, etc., before doing the thoracoplasty.

\section{Treatment of Group D}

(LUNG LESION AND PYOGENIC INFECTION BOTH PRESENT)

The patients of this group have the worst prognosis of all. The survivors, with few or no exceptions, will require a total thoracoplasty. The preliminary treatment is much the same as in Group C, but once the secondary infection is under control the thoracoplasty should be done as soon as possible, lest the lung should start to expand. If the patient remains too ill for thoracoplasty, permanent drainage and irrigation is all that is left to do.

\section{Aspiration and Air Replacement}

This can be done through one needle with two taps, or through two needles-one for the withdrawal of the fluid and the other for the running in of the air or gas.

\section{Pleural Lavage}

This can be done through one needle only if the cavity is small; some of the effusion is first withdrawn, and then a lesser amount of the irrigating fluid is run in. The process is repeated until the cavity is practically empty. If the cavity is large two needles should be used and, after aspirating, the cavity should be filled with the irrigating fluid from below upwards and this repeated until the return flow is clear. If lavage is successful the effusion becomes thinner and less infective, and gradually disappears.

Various fluids have been recommended for irrigations: (1) Dakin's solution is particularly useful when the pleura is thickened and there is severe secondary infection; (2) ordinary salt solution is used when the pleura is relatively thin, and especially if there is a bronchial fistula and irrigation is still wanted ; (3) dyes sometimes seem to do good-for example, 0.1 per cent. gentianviolet, or 0.2 per cent. methylene-blue, or 2 per cent. cresyl-violet.

After the irrigation a small amount of a stronger concentration may be instilled and left in-for example,
$20 \mathrm{c} . \mathrm{cm}$. of 1 per cent. gentian violet, or $20 \mathrm{c} . \mathrm{cm}$. of 2 per cent. methylene-blue in absolute alcohol if tubercle bacilli are found in the effusion; this should not be repeated more than once, as it sometimes causes a bloody exudation.

If the effusion is too thick to aspirate it may be made more fluid by means of certain modifying agents. There are three of these. (1) Gauvain's solution: guaiacol 2 parts, creosote 2 parts, iodoform 5 parts, ether 10 parts, and olive oil 100 parts. Twenty c.cm. are injected and left for twenty-four hours. The procedure is repeated, if necessary, until aspiration becomes possible. Oxygen or air replacement makes it easier. (2) Chlorazone or chloramine-T : 30 to $50 \mathrm{c} . \mathrm{cm}$. of a 0.5 per cent. solution is left in for twelve to eighteen hours and then aspirated with the effusion. (3) Pepsin solution. This consists of pepsin 20 parts, carbolic acid 2 parts, hydrochloric acid 2 parts, and distilled water to 400 parts ; 50 to $100 \mathrm{c} . \mathrm{cm}$. are left in for six to twelve hours.

\section{Oleothorax}

The chief indications for oleothorax are: (1) recurrent purulent effusion when the lung is diseased and our aim is to keep it collapsed, or if the lung tends to expand in spite of air refills; $(2)$ as a preliminary to drainage of a secondary infected empyema; (3) a small bronchial fistula; (4) severe toxaemia, whether from a pure tuberculous or from a mixed infection.

For these indications we use 5 per cent. gomenol in olive oil. An initial testing dose of $5 \mathrm{c.cm}$. only is given, and if there is no reaction several hundred cubic centimetres may be injected. Even if it fails to cure, it often makes the subsequent thoracoplasty safer. But it can be dangerous. It may make the lung condition worse, or it may cause a large pleural effusion, and although it may cure a bronchial fistula it may cause one if the empyema walls are stiff and the intrapleural pressure rises so much that the oil bursts through the lung.

\section{Intercostal Drainage}

The main use of drainage is to get the patient into better condition for more radical treatment. The chief indications are: secondary infection not controlled by aspiration, lavage, and air replacement; a bronchial fistula that does not close soon, especially if oleothorax has been tried; and rapid recurrence of effusion in spite of treatment by aspiration, etc. As a rule it is best to avoid drainage when there is still no secondary infection, but where the patient is under strict supervision and the empyema is of long standing, air-tight intercostal drainage may cure it when other means have failed. With proper care the drainage may be maintained for months without secondary infection occurring, and it may make an ill patient well enough for a thoracoplasty if it is required.

\section{Rib Resection and Drainage}

This is required when the more conservative measures have failed. The resection should be done in front of the anterior axillary line in order that there will be the clean operation field for the thoracoplasty that most of these patients will need.

\section{Phrenic Paralysis}

The chief use of phrenic paralysis in tuberculous empyema is to supplement other measures such as thoracoplasty or oleothorax. As it reduces the size of the hemithorax it may be beneficial in all groups of empyema. A persistent cutaneous sinus near the diaphragm may close after phrenic avulsion when other measures, such as cauterization, have failed. It also relieves symptoms 
due to involvement of the nerve and diaphragm in the inflammatory reaction, such as paroxysmal cough and pain.

\section{Thoracoplasty}

This is especially indicated when, first, the lung is diseased, particularly if there is cavitation or much fibrosis ; and, secondly, when the empyema cavity persists in spite of less radical treatment.

As the chest wall is rigid and thickened as the result of the empyema, it is necessary to remove large sections of the ribs in order to get the two visceral surfaces into contact, for the adhesion of these two layers is the essence of the operation. The operation consists of removal of the whole of the first rib, most of the second and third, and half to three-quarters of the fourth to the tenth ribs. It is done in two or more stages, with intervals of fourteen days or longer, according to the patient's condition. Between stages, and after the operation is completed, it is important to remove any effusion. To assist the falling in of the chest wall we try to delay the regeneration of the ribs by painting the rib beds with 10 per cent. formalin or with Zenker's fluid (potassium chromate 2.5 parts, sodium sulphate 1 part, corrosive sublimate 5 parts, glacial acetic acid 5 parts, and water 100 parts). The operation is begun from above and is usually supplemented by a phrenic avulsion. If the cavity is very large and the chest wall unusually thickened, the transverse processes may be removed with the corresponding ribs. After the operation the patient must wear a special thoracoplasty belt to maintain compression of the diseased side.

\section{Other Plastic Operations}

1. Where there is a large cavity or much calcification of the wall a preliminary modified pneumolysis may prove useful. One rib only is resected and the rest are stripped but left in place; the space between the ribs on one side and the intercostal structures and the periosteum on the other is packed with gauze, thus pushing the soft parts of the chest wall towards the mid-line, and the superficial tissues are sutured over the gauze pack. This is removed in ten days, and the wound is closed without drainage.

2. If the lung is completely collapsed and the walls of the cavity are very thick and there is a fistula, still more radical operation may be called for, such as complete decostalization, with removal of parietal pleura, intercostal tissues, and periosteum, and cauterization of the bronchial fistula. Decortication of the visceral pleura is contraindicated in tuberculous empyema.

3. Instead of excising the outer wall of the pleural cavity after the rib resections, the surgeon may make a flap from it so that it can be swung on to the visceral wall and kept in close contact by a pack, over which the superficial tissues are sewn. The pack is removed in ten days. This operation has the same rationale as (1) above, and is more likely to be successful.

\section{Results of Treatment}

The mortality of tuberculous empyema is between 30 and 50 per cent. The chief causes of death are secondary infection and tuberculosis of the lungs; less common causes are tuberculous meningitis and generalized tuberculosis. The main operative risks, apart from shock, haemorrhage, etc., are aspiration bronchopneumonia and other chest complications. J. Alexander, in a series of seventy patients, of whom sixty-nine had secondary infection and/or disease in the corresponding lung, found that 61.4 per cent. were cured-that is, the empyema cavity was obliterated and there were no tubercle bacilli in the sputum, or there was no sputum at all. Deaths accounted for 32.9 per cent., mainly from advance of the pulmonary disease, and spread to the opposite lung. In forty-five of the seventy cases thoracoplasty was done, and 80 per cent. of this group were cured. Brian Taylor, at the Brompton Hospital, studied a series of seventynine patients with tuberculous empyema. Twenty-five were alive at the end of five years (31.6 per cent.), forty-six were dead (58.2 per cent.), and the rest were untraced. Thirteen had thoracoplasty and four were dead at the end of the five-year period, a mortality of 30.8 per cent.

\section{Illustrative Case Records}

I have not yet seen a patient belonging to Group Athat is, with tuberculous empyema without secondary infection and without disease of the corresponding lung. As an example of Group B-pulmonary tuberculosis is known to be present and the empyema is not secondarily infected:

W. B., a male aged 19, was considered to be suitable for thoracoplasty for simple tuberculous empyema and tuberculosis of the underlying lung. Unfortunately the opposite lung was also diseased, and the patient's condition after the first stage became so bad that the rest of the operation had to be postponed indefinitely. Aspiration and lavage are being done in the meantime in the hope that the patient will improve sufficiently to stand the completion of the operation.

As an example of Group C-mixed tuberculous empyema without gross evidence of pulmonary tuberculosis :

E. B., a lad aged 19, was admitted to hospital with a left pleural effusion apparently secondary to a recent acute influenza or pneumonia. Although the organisms found in it resembled pneumococci, the fluid was thin and turbid, and suggestive of a streptococcal infection. Aspiration was ordered as a preliminary to thoracotomy, in accordance with the well-known procedure for streptococcal empyema. Further bacteriological examinations of the aspirated fluid revealed tubercle bacilli. Aspiration and lavage resulted in apparent cure, and the patient was sent to a sanatorium for further observation.

As an example of Group D-pulmonary tuberculosis is known to be present and the empyema is secondarily infected :

J. B., a male aged 20, had a spontaneous pneumothorax which resulted in an infected tuberculous empyema. Tubercle bacilli were found in the sputum, and the other lung was seriously affected. The empyema was drained through an intercostal tube by siphonage and suction. The patient was desperately ill, but gradually improved, and in the end he survived a total thoracoplasty done in three stages, supplemented by phrenic paralysis.

I am indebted to Brompton Hospital for opportunities to study this malady, and other chest diseases, during my term as surgical clinical assistant last year, and especially to $\mathrm{Mr}$ Tudor Edwards, under whom I worked, for counsel and instruction on all occasions, and for the case records quoted. I am also grateful to the other surgeons of Brompton Hospital and to Mr. Morriston Davies, all of whom generously gave me of their knowledge and experience.

It has been decided to hold at Royat every third or fourth year an international conference on some physiological, pathological, or therapeutic aspect of cardiovascular disease. The first of these conferences will be held during Whitsuntide, 1936, when the lecturers will be: Professors Heymans and Brouha, on vascular tonus; Professor Riser, on vascular spasm in the brain; Professors Leriche and Fontaine, on vascular spasms in the limbs; Professor Marañon, on vascular spasms associated with endocrine disturbances; and Professor Loeper, on the therapeutics of vascular spasms. The papers will appear afterwards in book form. 